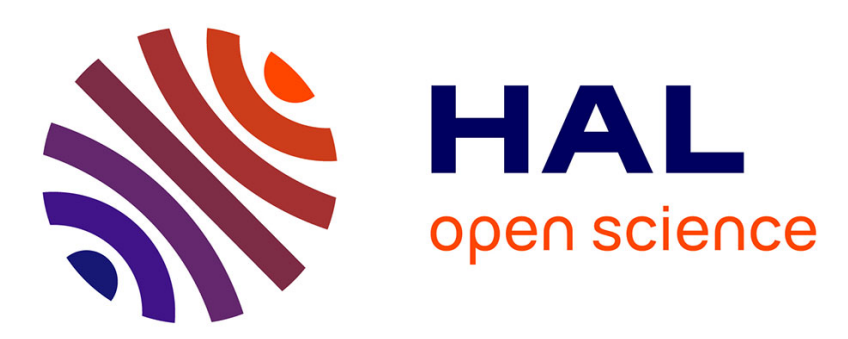

\title{
Traduire politiquement le soulèvement de mai 2021 : les défis du mouvement national palestinien
}

\author{
Nicolas Dot-Pouillard
}

\section{To cite this version:}

Nicolas Dot-Pouillard. Traduire politiquement le soulèvement de mai 2021: les défis du mouvement national palestinien. Confluences Méditerranée , 2021, Pouvoir(s) en Palestine, 117. halshs-03465965

\section{HAL Id: halshs-03465965 \\ https://shs.hal.science/halshs-03465965}

Submitted on 4 Dec 2021

HAL is a multi-disciplinary open access archive for the deposit and dissemination of scientific research documents, whether they are published or not. The documents may come from teaching and research institutions in France or abroad, or from public or private research centers.
L'archive ouverte pluridisciplinaire HAL, est destinée au dépôt et à la diffusion de documents scientifiques de niveau recherche, publiés ou non, émanant des établissements d'enseignement et de recherche français ou étrangers, des laboratoires publics ou privés. 


\title{
Traduire politiquement le soulèvement de mai 2021 : les défis du mouvement national palestinien
}

\begin{abstract}
Nicolas Dot-Pouillard est Political Advisor au Center for Humanitarian Dialogue (HD, Genève), et Chercheur associé à l'Institut français du Proche-Orient (Beyrouth). Il réside au Liban, et travaille dans les camps de réfugiés palestiniens. Il a récemment coordonné, avec Véronique Bontemps, Jalal al-Husseini et Abaher El Sakka, l'ouvrage Penser la Palestine en réseaux, Diacritiques Editions et Presses de l'Ifpo, Beyrouth, Marseille, 2020.
\end{abstract}

NB : cet article pour Confluences Méditerranée est la version longue, remaniée et actualisée d'un papier publié dans la revue en ligne Orient XXI le 25 mai 2021.

Avec le cessez-le-feu du 21 mai 2021 entre le Hamas et Israël, une nouvelle période s'ouvre pour le mouvement national palestinien. Le Hamas sort renforcé de la confrontation militaire avec Israël : il a bénéficié du soutien de toutes les factions dans la bande de Gaza, en un véritable moment d'unité par les armes. Dans les rapports de force inter-palestiniens, il a maintenant une longueur d'avance sur un Fatah divisé, entre défense d'un appareil proto-étatique, l'Autorité nationale palestinienne (ANP), et tentation d'un retour à l'Intifada. Les défis n'en sont pas moins immenses pour le Hamas, tout comme pour l'ensemble des partis politiques palestiniens. La réforme de l'Organisation de libération de la Palestine (OLP) attend encore. Le temps long de la réconciliation interpalestinienne contraste avec les attentes pressées du soulèvement de mai 2021, mobilisant en un moment décolonial inédit tout autant les Palestiniens des Territoires occupés et de la diaspora que ceux détenteurs de la citoyenneté israélienne. La structuration sur le long terme de la révolte palestinienne du printemps 2021 n'est pas acquise.

\section{La division palestinienne toujours recommencée}

À la fin du mois d'avril 2021, le spectre de la division inter-palestinienne refait surface. Le président de l'Autorité nationale palestinienne (ANP), Mahmoud Abbas, annule les élections législatives qui devaient se tenir au mois de mai, alors même que 36 listes ont été validées par la Commission centrale des élections, et que $93 \%$ des Palestiniens en âge de voter se sont inscrits sur les listes électorales. En arguant de l'impossibilité de tenir des élections à Jérusalem-Est, du fait d'un blocage israélien, le président de l'Autorité nationale palestinienne a remis en cause plusieurs mois de négociations inter-palestiniennes tenues en Cisjordanie, à Gaza, et dans l'ensemble de la région. La fragile réconciliation inter-palestinienne actée à Beyrouth par l'ensemble des factions membres et non-membres de l'Organisation de libération de la Palestine (OLP), en septembre 2020, validée lors d'une série de rencontres palestiniennes tenues au Caire et à Istanbul les mois suivants, tient en un simple agenda : organiser des élections législatives au printemps dans les Territoires occupés, suivies de présidentielles, puis renouveler le Conseil national palestinien (CNP) de l'OLP, censé représenter l'ensemble des Palestiniens des Territoires occupés et de la diaspora. Cette 
réconciliation nationale a un parrainage qatari, turc et égyptien, trois pays engagés dans les pourparlers inter-palestiniens. Elle bénéficie d'un réchauffement des relations diplomatiques entre le Caire, Doha et Istanbul, au cours des années 2020 et 2021 . Cette réconciliation nationale a aussi ses maîtres d'œuvre : Jibril Rajoub, secrétaire du Comité central du Fatah, et Salah al-Arouri, vice-président du Bureau politique du Hamas.

Le véritable motif de l'annulation des élections par Mahmoud Abbas tient plus aux divisions de son parti, le Fatah, mal en point: en avril, trois listes se réclament de l'héritage du président Yasser Arafat. La première, « officielle », est conduite par Mahmoud al-Alloul, vice-président du Fatah. La seconde liste est emmenée par Nasser Qudwa ${ }^{1}$, neveu de Yasser Arafat, expulsé du Comité central du Fatah au mois de mars, et par l'avocate Fadwa Barghouti, membre du Conseil révolutionnaire du Fatah, l'épouse du député Marwan Barghouti. Ce dernier, détenu par les Israéliens depuis 2002, souhaitait se présenter aux élections présidentielles palestiniennes: populaire, il aurait pu sans nul doute les remporter face à un Mahmoud Abbas à la légitimité déclinante. Son élection serait un cauchemar pour les Israéliens, confrontés à un scénario à la Nelson Mandela.

La troisième liste, enfin, est parrainée par Muhammad Dahlan et par son FatahCourant réformiste (Tyar al-islahi) : ancien homme fort de la bande de Gaza, expulsé du Fatah en 2011, il est aussi le commis-voyageur de la diplomatie des Emirats arabes unis, où il réside. Il a opéré une réconciliation avec le Hamas dans la bande de Gaza, qui s'est traduite récemment par le retour de Rachid Abou Chabak, l'un de ses proches et membre du Fatah-Courant réformiste, dans la bande de Gaza, après 15 ans d'exil. Pour le Hamas, le rapprochement avec Muhammad Dahlan est un choix pragmatique : le mouvement islamiste cherche à miner Mahmoud Abbas, tout autant qu'à desserrer l'étaux israélien sur la bande de Gaza. En effet, de bonnes relations avec les Emirats impliquent de meilleurs liens avec les services de sécurité égyptiens, qui tiennent les clés de la frontière sud de Gaza. Quant aux divisions électorales du Fatah, elles ont leurs raisons d'être. Le parti est à bout de souffle. Depuis les années 1990, le Comité central et le Conseil révolutionnaire, les deux organes politiques principaux du Fatah, se sont ossifiés et bureaucratisés, peinant à intégrer les jeunes cadres. Par ailleurs, ses membres vieillissants représentent moins la diaspora et la bande de Gaza que la Cisjordanie, siège de l'ANP2 ${ }^{2}$. La problématique de la construction d'un appareil étatique sans État sur une portion très réduite des Territoires occupés de 1967, et une pratique autoritaire du pouvoir confondant souvent cadres dirigeants du mouvement et structures de direction de l'OLP et de l'ANP, ont peu à peu miné le Fatah.

\footnotetext{
${ }^{1}$ Nasser Qudwa était également président de la Fondation Yasser Arafat. Il a été révoqué de ce poste en mars 2021 par le président de l'ANP, Mahmoud Abbas.

2 Nicolas Dot-Pouillard et Xavier Guignard, « Un congrès pour rien ? L'avenir incertain du Fatah de Mahmoud Abbas », Orient XXI, 3 janvier 2017, https://orientxxi.info/magazine/l-avenirincertain-du-fatah-de-mahmoud-abbas, 1636
} 
Au début du mois de mai 2021, l'ultime tentative de Mahmoud Abbas pour réconcilier le Hamas et le Fatah échoue. Il envoie alors son fidèle assistant, Hussein al-Cheikh, membre du Comité central du Fatah, au Qatar. Il s'agit alors de convaincre Doha de pousser le Hamas à un compromis : à défaut d'élections, un gouvernement d'union nationale pourrait être formé. Le Qatar n'insiste pas, le Hamas dénonce la manœuvre du président palestinien, et refuse le compromis, se posant en premier démocrate de la scène politique palestinienne : ce sera des élections ou rien.

Dès lors, la scène politique palestinienne n'inquiète plus les Israéliens. Le mouvement national palestinien est de nouveau divisé après les quelques tentatives de réconciliation de l'automne et de l'hiver 2020. Les élections législatives et présidentielles sont annulées, écartant toute possibilité de victoire législative du Hamas ou d'élections de Marwan Barghouti, potentiel présidentprisonnier. Il n'y aura pas non plus de renouvellement du Conseil national de l'OLP. Mahmoud Abbas est un président d'autant plus faible que son parti est de plus en plus divisé. L'affaiblissement du soutien arabe à la cause palestinienne peut se poursuivre dans la continuation des accords de normalisation de 2020 et de 2021 entre Israël, les Emirats, Bahreïn, le Soudan et le Maroc. La colonisation peut encore s'accélérer. Le gouvernement de Benjamin Netanyahou a les coudées franches : il n'anticipe pas une réponse politique palestinienne d'ampleur, alors que les forces armées israéliennes envahissent l'esplanade des Mosquées à Jérusalem, en plein mois de Ramadan, et que les expulsions de familles palestiniennes dans les faubourgs de Cheikh Jarrah et de Silwan se multiplient.

\section{Le Hamas : l'unité par les armes}

Le 10 mai 2021, Abou Obeida, le porte-parole des Brigades Ezzedin al-Qassam, la branche armée du Hamas, annonce qu'à six heures du soir, une première salve de roquettes sera lancée sur Israël si les forces armées israéliennes ne se retirent pas de l'Esplanade des mosquées, ainsi que du quartier de Cheikh Jarrah, à Jérusalem-Est. Dix minutes exactement avant la fin de l'ultimatum, les premières roquettes sont tirées de la bande de Gaza. Le Hamas prend ainsi l'initiative politique et impose son agenda aux Israéliens. Il s'agit d'un pari certes risqué car les bombardements israéliens sur la bande de Gaza, du 10 au 21 mai, tout autant qu'ils touchent majoritairement des civils, peuvent mettre en péril ses cadres et son arsenal balistique, censé quelque peu rééquilibrer les termes d'une guerre coloniale et asymétrique. Le Hamas souhaite toutefois imposer une nouvelle équation aux Israéliens, en reliant politiquement et symboliquement, à défaut de toute continuité territoriale, la bande de Gaza à Jérusalem. Ses revendications ne sont plus centrées sur la levée du blocus israélien de la bande de Gaza, comme par le passé mais se concentrent désormais sur la colonisation israélienne à Jérusalem. Un tournant fondamental dans la politique du mouvement islamiste s'est opéré. Le 14 mai 2021, Khaled Meshaal, président de la branche extérieure 
du Hamas, énonce les quatre conditions palestiniennes pour un cessez-le-feu ${ }^{3}:$ le retrait des troupes israéliennes de l'Esplanade des mosquées, le retrait des colons de Cheikh Jarrah, la libération de tous les prisonniers récemment incarcérés par les Israéliens, et l'arrêt des bombardements sur Gaza. En Cisjordanie, à Jérusalem et en Israël même, la guerre de Gaza ne met pas fin aux manifestations : au contraire, elles se multiplient en un effet d'entraînement de moins en moins contrôlable par les Israéliens. C'est inédit.

Le Hamas veut également être le parti de l'unité inter-palestinienne. C'est d'abord une unité par les armes, car dans le conflit qui oppose la bande de Gaza à l'armée israélienne, le Hamas n'est pas seul. Il bénéficie de l'appui politique et militaire de tous les partis palestiniens à Gaza. Sa branche militaire dispose certes de la capacité balistique la plus efficace, atteignant désormais Tel-Aviv, mais elle fait aussi partie d'une Chambre d'opération militaire commune (Ghurfat al-mushtaraka) associant toutes les factions. Née en mai 2018, la Chambre d'opération commune a mené une série d'exercices militaires dans la bande de Gaza en décembre 2020. Constituée de 12 groupes armés", elle regroupe autant des formations islamistes (Hamas, Mouvement du Jihad islamique en Palestine), que des formations de gauche (les Fronts populaires et démocratique pour la libération de la Palestine-FPLP et FDLP), mais aussi quatre brigades affiliées... au Fatah (les Brigades Abdel Qader al-Husseini, Ayman alJaoudeh et al-Amoudi, et l'Armée de la tempête). Les capacités militaires de ces brigades, cachées sous terre dans un sinueux réseaux de tunnels - le «métro » ${ }^{5}$ de plusieurs centaines de kilomètres, ne sont certes pas équivalentes : mais elles sont complémentaires. Les missiles à longues portées du Hamas et du Jihad islamique paralysent les aéroports de Tel-Aviv et de Eilat pendant quelques jours, tandis que les brigades attachées au FPLP, au FDLP, aux Comités de résistance populaire ou au Fatah se concentrent sur des tirs de plus courtes portées, sur l'envoie de missiles antitanks de type « Kornet» ou de mortiers sur des patrouilles israéliennes le long de la bande de Gaza. Lorsque le 10 mai 2021, Abou Obeida, porte-parole de la branche armée du Hamas, donne un ultimatum à Israël pour retirer ses troupes de l'Esplanade des mosquées, il le fait au nom de

\footnotetext{
3 "Mesha'al yakshif Shurut Fasa'il al-Muqawama bi-Ghaza li-Waqf Itlaq an-Nar" (Meshaal dévoile les conditions des factions de la résistance à Gaza pour un cessez-le-feu), Watan Voice, 14 mai 2021, https://www.alwatanvoice.com/arabic/news/2021/05/14/1414208.html

${ }^{4}$ La Chambre d'opérations commune regroupe les Brigades Ezzedin al-Qassam (Hamas), les Brigades al-Quds (Mouvement du Jihad islamique en Palestine), les Brigades Abou Ali Mustapha (Front populaire pour la libération de la Palestine-FPLP), les Brigades de la résistance nationale (Front démocratique pour la libération de la Palestine-FDLP), les Brigades du Martyr Jihad Jibril (Front populaire pour la libération de la Palestine-Commandement général), les Brigades des Martyrs d'al-Aqsa- groupe du Martyr Ayman al-Jaoudeh (Fatah), les Brigades Abdel Qader alHusseini (Fatah), les Brigades des Moujahidins, les Brigades des Partisans (Parti des Libres palestiniens), l'Armée de la tempête (Fatah), les Brigades Nasser Saleh al-Din (Comités de résistance populaire), les Brigades des Martyrs d'al-Aqsa- groupe al-Amoudi (Fatah).

5 Certains détails du fonctionnement du « métro » souterrain du Hamas et des factions palestiniennes ont été révélés par le responsable du Mouvement de la résistance islamique dans la bande de Gaza, Yahia Sinwar, lors de sa conférence de presse du 26 mai 2021. De son côté, le premier Ministre israélien Benjamin Netanyahou revendique la destruction de 100 kilomètres du « métro » palestinien lors des différentes phases de bombardement de l'armée israélienne.
} 
"la direction de la résistance de la Chambre d'opération commune », et non du mouvement islamique.

Être le garant de l'unité nationale et territoriale palestinienne est aussi affaire de symbole fort: une fois les bombardements terminés, le Hamas cherche à s'inscrire dans l'héritage de son frère ennemi, le Fatah. Le 22 mai 2021, le dirigeant du Hamas dans la bande de Gaza, Yahia Sinwar, parcourt des rues détruites à la surprise de toutes et tous, alors que les Israéliens se sont promis de l'exécuter - et ont bombardé sa demeure quelques jours auparavant. Dans les décombres, il rend un hommage improvisé, devant caméras et Smartphones allumés, au président Yasser Arafat, et entraîne une foule galvanisée à reprendre un ancien slogan du leader de l'OLP : « al-Quds, Rayhin, Shuhada bi-l-Malayin » ( à Jérusalem, nous allons martyrs par millions). La scène est immédiatement répercutée sur tous les réseaux sociaux palestiniens, de Facebook à Télégram, en passant par Twitter et TikTok. Quatre jours plus tard, lors d'une conférence de presse, il rend de nouveau hommage à Yasser Arafat, s'adressant directement au défunt par l'usage de la deuxième personne du singulier: le lendemain, l'ancienne épouse de Yasser Arafat, Soha, remercie chaleureusement le leader gazaoui du Hamas pour ses paroles. En se posant moins comme le leader d'un mouvement islamiste que comme l'héritier revendiqué du leader historique de l'OLP, il mobilise tout autant un imaginaire national partagé qu'il joue sur les contradictions -réelles- d'un Fatah à bout de souffle.

\section{Le Fatah : un retour à l'Intifada ?}

Avant le 10 mai 2021, le mouvement national palestinien s'est fracturé sur le dossier des élections. À l'époque, les factions palestiniennes, à l'exception du Fatah, dénoncent la suspension du processus électoral par le président palestinien. À la mi-mai cependant, elles soutiennent toutes le Hamas face à Israël. En reliant Gaza à Jérusalem, à la Cisjordanie et aux territoires de 1948, le mouvement islamique ne veut plus apparaître comme le gérant perpétuel d'une bande de Gaza assiégé, mais comme le garant de l'unité nationale et territoriale palestinienne, alors même que l'Autorité nationale de Mahmoud Abbas est de plus en plus critiquée en Cisjordanie en raison notamment de sa participation à la coopération sécuritaire : le 14 mai, de jeunes palestiniens au sud de Naplouse ont incendié un poste de police palestinien, protestant contre la coopération sécuritaire entre Israël et l'ANP.

Le 15 mai 2021, dans une vidéo circulant sur les réseaux sociaux palestiniens, le vice-président du Fatah, Mahmoud al-Alloul, appelle les militants de son parti à participer à toutes les manifestations populaires à Jérusalem-Est et en Cisjordanie contre la colonisation israélienne. Le 18 mai, le mouvement de jeunesse (Shabiba) du Fatah mobilise ses militants lors des manifestations qui accompagnent la grève générale décrétée par les factions palestiniennes, les syndicats, les associations et les mouvements de jeunesse indépendants dans 
l'ensemble de la «Palestine historique », c'est à dire comprenant les Palestiniens des Territoires occupés et ceux détenteurs de la citoyenneté israélienne. La veille, au soir, la branche armée du Fatah, les Brigades des Martyrs d'al-Aqsa ${ }^{6}$ (BMA), défile dans les rues de Ramallah, pour la première fois depuis 16 ans. Le lendemain, elles s'accrochent à l'armée israélienne, à al-Bireh, non loin de Ramallah. Mahmoud Abbas s'était pourtant promis d'interdire les activités des BMA, depuis son accession à la présidence palestinienne, en janvier 2005. Son parti semble hors de contrôle. Sa base populaire est sensible à une Intifada naissante.

Les initiatives militaires du Hamas ont un effet d'entraînement sur le Fatah. Lorsque les Israéliens cessent de bombarder la bande de Gaza, le 21 mai, la branche est du Fatah dans la bande de Gaza publie un communiqué appelant à l'unité nationale et à continuer le soulèvement dans les Territoires occupés de 1967 et de 1948 (Israël). La base du Fatah pourrait être également sensible aux appels du député emprisonné Marwan Barghouti à poursuivre et structurer le soulèvement en cours, et à réformer l'OLP : dans une lettre de prison publiée le 15 mai, il rappelle que le Fatah est le «protecteur du projet national révolutionnaire palestinien », mais il appelle également à intégrer le Hamas et le Mouvement du Jihad islamique en Palestine (MJIP) au sein de l'OLP, "maison commune aux Palestiniens $» 7$. Deux jours après la publication de cette lettre, il est mis en isolement carcéral par les Israéliens. Des cadres du Fatah ou des démissionnaires du mouvement de plus en plus opposés au président peuvent aussi, début juin, signer une pétition d'universitaires, d'écrivains et d'intellectuels palestiniens appelant officiellement à la démission de Mahmoud Abbas $^{8}$, un pas que même le Hamas ou le Mouvement du Jihad islamique ne sauraient franchir.

\footnotetext{
6 Sur l'histoire des Brigades des Martyrs d'al-Aqsa, voir Minas Ouchaklian, « Les groupes armés du camp de Balata (Cisjordanie). Mobilisation, révolte et économie parallèle à l'ombre des réseaux du Fatah », in Penser la Palestine en réseaux, sous la direction de Véronique Bontemps, Nicolas Dot-Pouillard, Jalal al-Husseini et Abaher El Sakka, Diacritiques éditions et Presses de l'Ifpo, Beyrouth, Marseille, 2020, pp 124-151, et Jean-François Legrain, Internet et histoire. Les Brigades des Martyrs d'al-Aqsa. Les pages internet comme sources de l'histoire du temps présent, Maison de l'Orient et de la Méditerranée, Lyon, 17 juin 2004, https://www.mom.fr/guides/aqsa/aqsa.htm
}

7 «Marwan Barghouti yad'u ila-l-Iltifaf hawal-l- Muqawama wa-l-Tamasuk bi-l-Wahda » (Marwan Barghouti appelle au rassemblement autour de la résistance et à tenir bon dans l'unité), Watan Voice, 15 mai 2021, https://www.alwatanvoice.com/arabic/news/2021/05/15/1414281.html

\footnotetext{
${ }^{8}$ La pétition a été signée par des universitaires palestiniens comme Sari Hanafi (Liban) et Khaled Hroub (Cambridge University), par des membres du Conseil national de l'OLP comme Salman Abu Sitta ou Saber Aref, ainsi que par des membres présents ou démissionnaires du Fatah, dont Mueen al-Taher, ancien responsable de la Brigade étudiante du Fatah pendant les guerres du Liban. Le texte et les signataires de la pétition sont disponibles à l'adresse suivante : https://www.change.org/p/\%D8\%A7\%D9\%84\%D8\%B4\%D8\%B9\%D8\%A8\%D8\%A7\%D9\%84\%D9\%81\%D9\%84\%D8\%B3\%D8\%B7\%D9\%8A\%D9\%86\%D9\%8A\%D8\%B9\%D8\%B2\%D9\%84-\%D9\%85\%D8\%AD\%D9\%85\%D9\%88\%D8\%AF\%D8\%B9\%D8\%A8\%D8\%A7\%D8\%B3-\%D9\%85\%D9\%86-
} 
La direction du Fatah a bien conscience qu'un changement d'époque s'est produit. Jibril Rajoub, secrétaire du Comité central du Fatah et artisan du dialogue national avec le Hamas ouvert à Beyrouth en septembre 2020, admet, lors d'un interview accordée à une chaîne télévisée saoudienne le 19 mai, qu'une " nouvelle réalité » s'est imposée, et que «les choses ne reviendront plus à ce qu'elles étaient avant le 10 mai 2021 », date du début de la bataille de Gaza ${ }^{9}$. Au sein du Fatah, la tentation d'un retour à l'Intifada est donc forte d'autant plus qu'il est maintenant complètement dépassé par le Hamas qui se pose en avantgarde de l'unité nationale et qui a réussi le tour de force de relier politiquement et symboliquement la bande de Gaza à Jérusalem. Néanmoins, le Fatah dépend encore financièrement et politiquement de l'Autorité nationale palestinienne, laquelle est tenue à bout de bras par les principaux états occidentaux. En Cisjordanie, des membres du Fatah, cumulant affiliation partisane et appartenance aux appareils de police et de renseignements palestiniens, sont engagés dans la coopération sécuritaire avec Israël. À partir du 21 mai 2021, date de la fin des bombardements, les arrestations opérées par les services de l'Autorité contre des activistes islamistes ou de gauche en Cisjordanie ont repris comme par le passé. La concurrence avec le Fatah-Courant réformiste de Muhammad Dahlan, proche des Émirats, soude la majorité du Comité central du Fatah autour du président Mahmoud Abbas. La page de l'Autorité nationale palestinienne n'est donc pas complètement tournée, mais les tensions apparues au sein du Fatah lors du processus électoral avorté du printemps 2021 se sont accrues avec la dynamique du soulèvement.

\section{Traduire politiquement le soulèvement}

Le Hamas est maintenant à l'offensive politique et diplomatique et mène le jeu en Palestine. La médiation égyptienne pour un cessez-le-feu avec Israël lui donne une stature régionale. Le Hamas et la Chambre d'opérations communes n'ont certes pas gagné sur leurs trois revendications majeures: l'arrêt des provocations israéliennes sur l'esplanade des Mosquées, le retrait des colons de Cheikh Jarrah, et la libération des prisonniers politiques. Néanmoins, ils pensent avoir quelques acquis : les Égyptiens tentent de relancer désormais les négociations entre Israël et le Hamas sur le dossier des prisonniers; sur le dossier de Jérusalem, les Israéliens tiennent plus compte que par le passé des lignes rouges du Hamas: le 15 juin 2021, les Israéliens assurent aux négociateurs Egyptiens que la " Marche du drapeaux », organisée par l'extrêmedroite israélienne à Jérusalem-Est, sera limitée en nombre de participants et ne franchira pas les limites symboliques de la porte de Damas- en échange de quoi

\%D9\%85\%D9\%86\%D8\%A7\%D8\%B5\%D8\%A8\%D9\%87-

\%D8\%A7\%D9\%84\%D9\%82\%D9\%8A\%D8\%A7\%D8\%AF\%D9\%8A\%D8\%A9

9 «Al-Rajoub yakshif : Itifaq murtaqab li-Waqf Itlaq al-Nar khilal al-Sa'at al-muqbila » (Rajoub révèle qu'un accord de cessez-le-feu est attendu dans les prochaines heures), Watan Voice, 19 mai 2021, https://www.alwatanvoice.com/arabic/news/2021/05/19/1414943.html 
le Hamas s'engage à ne pas reprendre de tirs de roquettes - une négociation dans laquelle l'Autorité nationale palestinienne et le Fatah ont été les grands absents; surtout, en paralysant l'économie et la circulation des biens et des personnes pendant onze jours en Israël, sans que le système israélien de missiles anti-missiles - le «dôme de fer»- ne puisse réellement enrayer l'envoie de roquettes palestiniennes, le Hamas a mené une guerre courte et explicite. Son message a été bien compris par les Égyptiens, mais aussi par les Jordaniens et les Qataris, engagés dans les pourparlers de cessez-le-feu avec Israël. Dans les rapports de pouvoirs inter-palestiniens, c'est moins l'Autorité nationale palestinienne qui compte désormais que le Hamas et ses alliés. Son agenda s'est imposé.

Tout en remerciant l'Iran pour son soutien "financier et militaire », le président du Bureau politique du Hamas, Ismaël Haniyeh, dans un discours prononcé le 21 mai 2021, appelle à "renforcer les relations avec la communauté internationale ", tout en prenant en compte de "grands changements dans les sociétés européennes et occidentales ${ }^{10}$. Les portes de Damas s'ouvrent de nouveau au Mouvement de la résistance islamique, comme semblent l'attester les plus récentes déclarations de Bashar al-Assad, après plusieurs années de brouilles dues au parti-pris du Hamas pour l'opposition syrienne lors de la guerre civile ${ }^{11}$. En même temps, le Hamas est désireux de s'ouvrir sur l'Union européenne et les Etats-Unis, comme il l'a fait par le passé avec la Russie ${ }^{12}$. La diplomatie du Hamas est multilatérale. Idéologiquement, en raison de son affiliation aux Frères musulmans, le Hamas peut compter sur un soutien financier turc et qatari, où réside une partie de sa direction extérieure. Militairement et politiquement, il est proche de l'Iran et du Hezbollah, en raison d'une longue histoire de coopération sécuritaire dans leur opposition commune à Israël. Pragmatiquement enfin, le Hamas a prouvé qu'il peut travailler avec la diplomatie et les services de sécurité égyptiens, l'Égypte servant de boîte aux lettres et de porte d'entrée sur les Etats-Unis et l'Union européenne.

\footnotetext{
10 «Haniyeh : al-Nasr sana'ahu al-Sha'ab al-filistini wa-l-muqawama wa lan natakhala 'an Iltizamatina » (Haniyeh : la victoire a été faite par le peuple palestinien et la résistance et nous n'abandonnerons pas nos engagements), al-Quds.net, 21 mai 2021, https://qudsn.net/post/184312/\%D9\%87\%D9\%86\%D9\%8A\%D8\%A9\%D8\%A7\%D9\%84\%D9\%86\%D8\%B5\%D8\%B1-\%D8\%B5\%D9\%86\%D8\%B9\%D9\%87\%D8\%A7\%D9\%84\%D8\%B4\%D8\%B9\%D8\%A8\%D8\%A7\%D9\%84\%D9\%81\%D9\%84\%D8\%B3\%D8\%B7\%D9\%8A\%D9\%86\%D9\%8A\%D9\%88\%D8\%A7\%D9\%84\%D9\%85\%D9\%82\%D8\%A7\%D9\%88\%D9\%85\%D8\%A9\%D9\%88\%D9\%84\%D9\%86-\%D9\%86\%D8\%AA\%D8\%AE\%D9\%84\%D9\%89\%D8\%B9\%D9\%86\%D8\%A7\%D9\%84\%D8\%AA\%D8\%B2\%D8\%A7\%D9\%85\%D8\%A7\%D8\%AA\%D9\%86\%D8\%A
} 7

11 Adnan Abu Amer, « Syria's Assad, Hamas Weight Rapprochment », Al Monitor, 29 mai 2021, https://www.al-monitor.com/originals/2021/05/syrias-assad-hamas-weigh-rapprochement

12 Leila Seurat, Le Hamas et le monde : la politique étrangère du mouvement islamiste palestinien (2006-2015), Préface de Bertrand Badie, CNRS Editions, Paris, 2015. 
Le Hamas a un défi : proposer des perspectives stratégiques et jouer un rôle moteur dans la réconciliation inter-palestinienne. L'unité dans la résistance armée à Gaza est une chose, la structuration sur le long terme d'une Intifada naissante chez l'ensemble des Palestiniens en est une autre. Pour le moment, de nombreux points d'interrogation subsistent sur le débouché politique $\mathrm{du}$ soulèvement: articulation de la lutte armée dans la bande de Gaza et des manifestations populaires en Cisjordanie et à Jérusalem; liens avec les Palestiniens détenteurs de la citoyenneté israélienne; ouverture sur une diaspora palestinienne fortement mobilisée au Liban et en Jordanie ; équilibre précaire à maintenir entre des puissances régionales aussi discordantes que l'Iran, l'Égypte et les pays du Golfe.

Trois chantiers internes attendent maintenant le Hamas et les factions palestiniennes. Le premier chantier est celui de la réforme de l'OLP. Si la question des élections législatives palestiniennes dans les Territoires occupés semble pour le moment compromise, celle d'une transformation de la centrale palestinienne fait consensus parmi les factions. Déjà, en mai 2011, le Hamas et le Fatah s'accordaient sur la mise en place d'un Cadre de direction transitoire de l'OLP, gelé depuis, intégrant le Hamas et le Mouvement du Jihad islamique en Palestine dans ses rangs. En juin 2021, une nouvelle série de pourparlers interpalestiniens au Caire remet encore une nouvelle fois à l'ordre du jour la réforme de l'OLP - les Egyptiens renoncent cependant à faire s'asseoir à la même table le Fatah et le Hamas, et se contentent de pourparlers bilatéraux avec chacune des formations. Les mécanismes de renouvellement de l'OLP restent toutefois encore à définir car ils ne sont pas seulement organisationnels - l'intégration du Hamas et du MJIP au sein de la centrale palestinienne- mais également générationnels et programmatiques. Les jeunes manifestants de Jérusalem ou de Ramallah n'ont pas même connu la seconde Intifada de septembre 2000. La colonisation des Territoires occupés a quant à elle rendu la perspective de deux États israéliens et palestiniens côte à côte de plus en plus impossible matériellement - même si le Hamas se dit toujours prêt à accepter une «trêve " (Hudna) de long terme sur les frontières de 1967. Enfin, depuis la conférence inter-palestinienne de Beyrouth, en septembre 2020, les factions s'interrogent sur le renouvellement du Conseil national de l'OLP. En principe, celui-ci doit être électif, mobilisant les millions de Palestiniens des Territoires occupés et de la diaspora mais matériellement, la tâche apparaît impossible car les registres de l'UNRWA des Palestiniens réfugiés au Liban ne correspondent pas à ceux du recensement gouvernemental libanais de $2017^{13}$ et une partie des réfugiés palestiniens de Syrie ont été déplacés depuis 2011. Une solution de rechange à un mécanisme électif consisterait à accorder des sièges au Conseil national palestinien de l'OLP en fonction d'un consensus préalable entre les factions, ou sur la base des résultats électoraux d'élections législatives palestiniennes dans les Territoires - qui ont été annulées.

Le second chantier est celui de la réconciliation entre le Hamas et le Fatah. Or, avec l'annulation des élections législatives du printemps 2021, tout comme avec

\footnotetext{
13 Nicolas Dot-Pouillard, « Jouer le jeu ? Les Palestiniens du Liban, entre marginalisation sociale et rhétorique de l'officiel », in Fragments palestiniens. Pouvoir, territoire et société, sous la direction de Xavier Guignard et Leyla Seurat, Revue des mondes musulmans et de la Méditerranée, numéro 147, Presses de l'Université de Provence, 2020, pp 70-87.
} 
le soulèvement du mois de mai et la guerre à Gaza, les relations entre les deux mouvements se sont singulièrement tendues - en dépit des hommages répétés de Yahia Sinwar à Yasser Arafat, et de la tentation d'un retour à l'Intifada du Fatah. En Cisjordanie, les services de l'Autorité pourchassent de nouveau des membres du Hamas. À Gaza, le Hamas a également procédé à des arrestations de certains membres du Fatah. Le 28 mai 2021, le secrétaire-général du Comité central du Fatah, Jibril Rajoub, parrain de la réconciliation inter-palestinienne de septembre 2020, signe une "Charte d'honneur» (Mithaq Charaf) avec des membres du Hamas en Cisjordanie, en présence du président de la Commission indépendante des Droits de l'homme, Omar Dweik. La Charte appelle à cesser les arrestations respectives avec un succès à cette heure limité. Or, sans un minimum de réconciliation entre le Hamas et le Fatah, c'est la réforme même de l’OLP qui est impossible.

Le troisième chantier est celui de la structuration du soulèvement de mai 2021 car le cessez-le-feu entre le Hamas et Israël n'a pas mis fin aux manifestations en Cisjordanie, et ne présage pas d'une accalmie de long terme à Gaza ou en Israël où les Palestiniens de 1948 font face depuis plusieurs semaines à une vague d'arrestation sans précédent. Lors d'un rassemblement du Front populaire pour la libération de la Palestine tenue à Gaza, le 2 juin 2021, Jamil Mazhar, membre du Bureau politique du FPLP, appelle urgemment à la formation de « Comités de protections populaires » en Cisjordanie contre la colonisation sur le modèle de la première Intifada de décembre 1987. Elle avait, à l'époque, au-delà de la spontanéité populaire née dans la bande de Gaza, son débouché politique: la naissance d'une Direction nationale unifiée de l'Intifada et le symbole fort de la Déclaration d'indépendance palestinienne du 15 novembre 1988, votée par le Conseil national de l'OLP. La seconde Intifada de septembre 2000 butta en l'occurrence sur la division entre le Fatah et le Hamas, et sur l'absence d'unité et de programme de transition inter-palestinien vers un État souverain.

Pour le mouvement national palestinien, il y a donc un risque que le retour à la division entre le Hamas et le Fatah et l'absence de réconciliation interpalestinienne apparaissent en complet décalage avec un soulèvement populaire historiquement inédit, mobilisant en même temps les Palestiniens des Territoires occupés et de la diaspora, tout comme ceux détenteurs de la citoyenneté israélienne. Le Hamas, les composantes du Fatah les plus opposées à Mahmoud Abbas et le des partis politiques palestiniens ont aujourd'hui un constat commun : une page s'est définitivement tournée sur les accords d'Oslo. Une nouvelle conjoncture politique s'est ouverte. La question est maintenant celle de leur coordination et de la traduction politique du soulèvement sur le long terme. De ce point de vue, deux modèles possibles apparaissent : celui de la première Intifada de décembre 1987, traduite à l'époque par la naissance d'une Direction nationale unifiée de l'Intifada et celui de septembre 2000, qui a échoué en raison des divisions inter-palestiniennes. Réforme de l'OLP, avenir de l'ANP, dialogue national, articulation entre lutte armée et manifestations populaires, définition de l'État palestinien à venir et de ses frontières : autant de débats inter-palestiniens qui prennent du temps. Ce temps long et patient de la stratégie est tributaire de logiques partisanes parfois opposées, de pesantes tractations d'appareils, mais aussi de véritables contraintes régionales et internationales 
dont les partis palestiniens tiennent aussi compte. Il contraste encore avec la spontanéité décoloniale du mois de mai 2021, vive, radicale et pressée, des manifestants palestiniens de Lod et de Haïfa, de Cheikh Jarrah ou de la diaspora. 